\title{
Research on Accuracy of Normal Moveout in Reflection Seismic Exploration by Cross Correlation Method
}

\author{
Yan Huang ${ }^{1}$, Hefeng Zhou ${ }^{1}$, and Xinwu Zeng ${ }^{1, *}$ \\ ${ }^{1}$ College of Meteorology and Oceanology, National University of Defense Technology, Changsha 410073, Hunan, China
}

\begin{abstract}
In this paper, we took the time-distance curve equation and the Normal Moveout (NMO) principle as breakthrough point. According to the forward seismic simulation model and the crosscorrelation principle in signal processing, we have convincingly studied the accuracy of NMO in simulation experiment. Firstly, the time-distance curve equation of single-layer reflection and the characteristics of cross-correlation are simply analysed. Then, we build the seismic forward model by Tesseral software, and accurate results are obtained after the simulation experiment is carried out. Finally, we get the conclusion by analysing and summarizing these results. The conclusion shows that the method effectively demonstrates the feasibility of the cross-correlation method in testing the accuracy of NMO, and provides a new idea for analysing the accuracy of velocity picking, even the accuracy of the underground model during petroleum exploration.
\end{abstract}

\section{Introduction}

Reflection seismic exploration [1-2] refers to the geophysical exploration method of inferring the nature and morphology of subsurface media by observing and analysing the response of solid or liquid media to artificially excited seismic waves (sound waves), which is based on the difference in elasticity and density of the subsurface medium. It is currently the main method for exploring mineral resources such as oil and natural gas in the world. With the advent of digital computers, the reflection seismic method entered the digital age in the mid-1960s. Digital technology has fundamentally revolutionized seismic data processing. Digitalization makes data representation flexible and facilitates largescale calculations, especially for seismic data processing with large data volumes.

The three major techniques of seismic processing are deconvolution, stack and migration imaging, while NMO [3-4] is one of the cores of the stack technique. The purpose is to eliminate the effect of offset to the traveling time of reflected wave, so that the signal of each receiver could be correctly stacked, thereby improving the signal-to-noise ratio (SNR). How to control the accuracy of NMO and ensure that the seismic simulation model conforms to the actual geological conditions has always been one of the hot spots and difficulties of reflection seismic exploration. Abedi [5] discussed the different application conditions of the NMO in the conventional time-distance curve and the special time-distance curve. Jianjun You [6] and others conducted the relative research on seismic NMO inphase axis under isotropic media, and proposed different processing methods for NMO under different offset conditions. Yangyang Zhao [7] also compared the accuracy of NMO under the condition of long offset in isotropic medium, and gave the optimal approximation of the time-distance curve in the case of long offset.

Cross-correlation [8] is a concept in signal analysis, which shows the degree of correlation between two time series, that is, the value of the description signal $x(t)$, $y(t)$ at any two different times $t_{1}, t_{2}$. When describing the correlation between two different signals, the two signals could be either random signal or deterministic signal. The meaning of the cross-correlation is that the infinite integral of the two functions after the process of complex conjugate, inverse translation and multiplying. In other words, the meaning of the cross-correlation of two functions is that the infinite integral of the first function after the process of complex conjugate and translation and multiplied by the second function. It could be shown that the two definitions are completely equivalent. Physically, the results of the cross-correlation operation reflect a measure of the similarity between the two signals.

\section{Forward simulation experiment}

This experiment is based on the principle of reflection seismic exploration and the definition of crosscorrelation function. Firstly, the experimental principle is briefly introduced in theory. Then the experimental parameters are determined according to the principle of easy processing and conformity with the actual situation, and we also established the forward model by Tesseral software. Finally, based on the forward model that is processed to obtain the shot record, velocity spectrum

* Corresponding author: xinwuzeng@nudt.edu.cn 
analysis and interactive picking root mean square (rms) velocity are conducted. In addition, recording the picked velocity value in order to prepare for the data processing in the following work.

\subsection{Experimental principle}

\subsubsection{Time-distance curve equation}

Figure 1 shows a schematic diagram of a two-layer medium, single reflection interface. Starting at a given middle point $\mathrm{M}$, along the ray from shot point $\mathrm{S}$ to depth point $\mathrm{D}$, and then back to the point $\mathrm{G}$ for a two-way travel time named $t_{x}$. Based on the Pythagoras theory, the equation that two-way travel time as a function for offset is

$$
t_{x}^{2}=t_{0}^{2}+\frac{x^{2}}{v^{2}(z)}
$$

As shown in Equation (1), the two-way travel time $t_{x}$ corresponding to the offset $x$, the overlay velocity value at the interface of the picked-up reflective layer is $v(z)$, and $t_{0}$ is the two-way travel time along the vertical path MD (zero offset), that is, the self-excited self-receiving time. Note that the projection of the depth point to the ground coincides with the middle point $\mathrm{M}$ along the orthogonal line of the reflection layer. Of course, this is only the case when the reflective layer is horizontal.

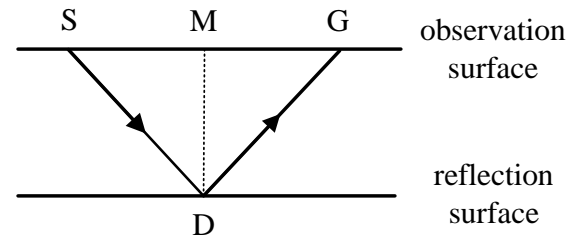

Fig. 1. Normal time difference geometry of a single horizontal reflective layer.

The difference between a two-way travel time on a given offset and a zero-offset travel time is called the amount of $\mathrm{NMO}$, expressed as $\Delta t$, and $\Delta t$ can be obtained based on (1), as shown in Equation (2).

$$
\Delta t=\operatorname{sqr} t\left[t_{0}^{2}+\frac{x^{2}}{v^{2}(z)}\right]-t_{0}
$$

Therefore, after we interactively pick up the velocity value, the $\Delta t$ could be obtained by inversely deriving from the equation (2), so that the deviation value of the NMO could be known after comparing with the actual data, and then the accuracy of velocity pickup could also be calculated.

\subsubsection{NMO and stack}

As shown in Figure 2, when the $\Delta t$ is calculated, the effect of offset on the arrival time of wave could be eliminated after the process of NMO. By stacking all the seismic data of the receivers after NMO, a specific stack for common middle point (CMP) is obtained. Therefore, seismic data could be stacked in phase. That is the purpose of NMO.

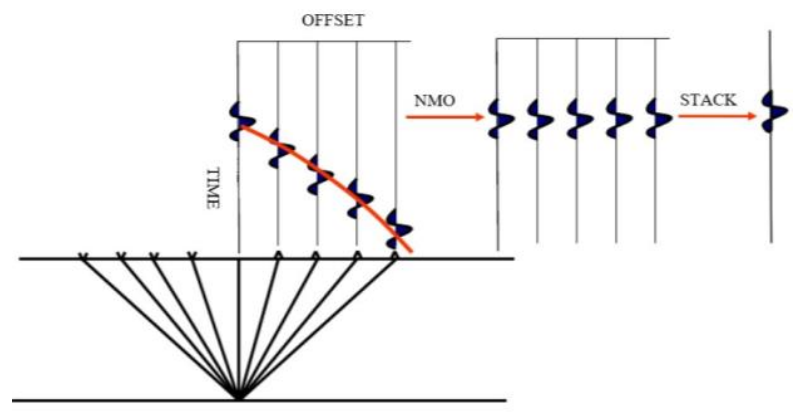

Fig. 2. Normal moveout and stack.

It has been studied that the value of NMO increases with the offset rises, and decreases with depth reduces. At the same time, the higher the velocity is, the smaller the NMO value is.

\subsubsection{Cross-correlation}

The cross-correlation of two functions $f(x)$ and $h(x)$ is defined by the infinite integral with the parameter $x$, that is

$$
\begin{aligned}
& R_{f h}(x)=\int_{-\infty}^{\infty} f^{*}\left(x^{\prime}\right) h\left(x^{\prime}+x\right) d x^{\prime} \\
& R_{f h}(x)=\int_{-\infty}^{\infty} f^{*}\left(x^{\prime}-x\right) h\left(x^{\prime}\right) d x^{\prime}
\end{aligned}
$$

Similar to convolution, the parameter $x$ and the integral variable $x^{\prime}$ are both real numbers, and the functions $f(x)$ and $h(x)$ could be real numbers or complex numbers.

Equation (3) shows that the cross-correlation of two functions means that the infinite integral of the two functions after the process of complex conjugate, inverse translation and multiplying. Equation (4) shows that the meaning of the cross-correlation of two functions is that the infinite integral of the first function after the process of complex conjugate and translation and multiplied by the second function. It could be shown that the two definitions are completely equivalent.

In particular, for the real functions $f(x)$ and $h(x)$, the correlation operation is equivalent to the area of the area surrounded by the overlapping portion and the horizontal axis formed by the relative translation of the two functions.

In order to simplify the calculation, the relevant operator symbol " $\otimes$ " is usually introduced, so that the cross-correlation of $f(x)$ and $h(x)$ could be expressed as Equation (5). Because it is satisfied with the exchange law, so written as Equation (6) as well.

$$
\begin{gathered}
R_{f h}=f(x) \otimes h(x) \\
R_{f h}=h(x) \otimes f(x)
\end{gathered}
$$

\subsection{Model establishment and velocity picking}

The basic parameters for establishing a two-layer horizontal medium model in Tesseral could be seen in Table 1. 
Table 1. Basic parameters.

\begin{tabular}{|c|c|}
\hline Parameter & value \\
\hline Shot type & single-side shot \\
\hline Wavelet frequency & $40 \mathrm{~Hz}$ \\
\hline wavelet type & Ricker \\
\hline Receiver number & 200 \\
\hline $\begin{array}{c}\text { Shot number } \\
\text { Distance between } \\
\text { receiver }\end{array}$ & 32 \\
\hline $\begin{array}{c}\text { Distance between } \\
\text { shot }\end{array}$ & $5 \mathrm{~m}$ \\
\hline Offset & $25 \mathrm{~m}$ \\
\hline sample rate & $4000 \mu \mathrm{m}$ \\
\hline
\end{tabular}

The depth of the reflective interface is $500 \mathrm{~m}$, and the medium velocity of the upper and lower layers are $2000 \mathrm{~m} / \mathrm{s}$ and $2500 \mathrm{~m} / \mathrm{s}$, respectively.

After building the model according to the above parameters, the calculation model and the generated velocity spectrum are shown in Figure 3.

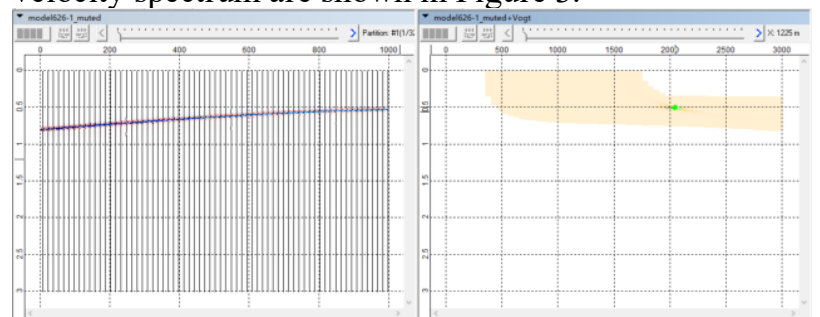

Fig. 3. Shot record map and velocity picking

According to the velocity picking principle, a picking velocity value is selected at the maximum stack amplitude, which is the stack velocity of the in-phase axis in the input CMP dataset. The low-amplitude horizontal trajectories on the velocity spectrum are the stack of the small offset components, and the highamplitude region is the stack of all the offset components. Therefore, we must retain the long offset data to ensure the resolution. Finally, the value of display velocity we pick is $2008.0 \mathrm{~m} / \mathrm{s}$.

\section{Experimental results and analysis}

Substituting the picked velocity value into the two-way travel time equation of the time-distance curve, $t_{0}$ could be calculated as $0.498 \mathrm{~s}$. The experiment plans to analyze the NMO accuracy at three locations with offsets of $x_{1}, x_{2}$ and $x_{3}$, the values correspond to $230 \mathrm{~m}, 500 \mathrm{~m}$, and $800 \mathrm{~m}$, respectively. In the shot record map, the coordinates of the three corresponding receivers are calculated to be $995 \mathrm{~m}, 825 \mathrm{~m}$, and $425 \mathrm{~m}$, respectively. Substituting these to Equation (1) could calculate the corresponding values of $t_{x}$ as $0.5110 \mathrm{~s}, 0.5568 \mathrm{~s}$, and
$0.6378 \mathrm{~s}$, respectively. Therefore, the corresponding NMO values $\Delta t$ could be calculated as $0.0130 \mathrm{~s}, 0.0588$ $\mathrm{s}$, and $0.1398 \mathrm{~s}$, respectively. The amount of delay of seismic waves between the offsets of $500 \mathrm{~m}, 800 \mathrm{~m}$ and the near offset $(230 \mathrm{~m})$ are calculated as $0.0458 \mathrm{~s}, 0.1268$ $\mathrm{s}$, respectively. These results could also be seen in Table 2 as follows.

Table 2. Experiment results

\begin{tabular}{|c|c|c|c|}
\hline Offset & Coordinate & $t_{x}$ & $\Delta t$ \\
\hline $230 \mathrm{~m}$ & $995 \mathrm{~m}$ & $0.5110 \mathrm{~s}$ & $0.0130 \mathrm{~s}$ \\
\hline $500 \mathrm{~m}$ & $825 \mathrm{~m}$ & $0.5568 \mathrm{~s}$ & $0.0588 \mathrm{~s}$ \\
\hline $800 \mathrm{~m}$ & $425 \mathrm{~m}$ & $0.6387 \mathrm{~s}$ & $0.1398 \mathrm{~s}$ \\
\hline
\end{tabular}

The data in 86th channel (the value of offset is $800 \mathrm{~m}$ ) and the data in 146th channel (the value of offset is 500 $\mathrm{m})$ in the shot record are extracted firstly, and the crosscorrelation processing is performed with the data in 200th channel (the value of offset is $230 \mathrm{~m}$ ) respectively by MATLAB software. The processing result is shown in Figure 4.

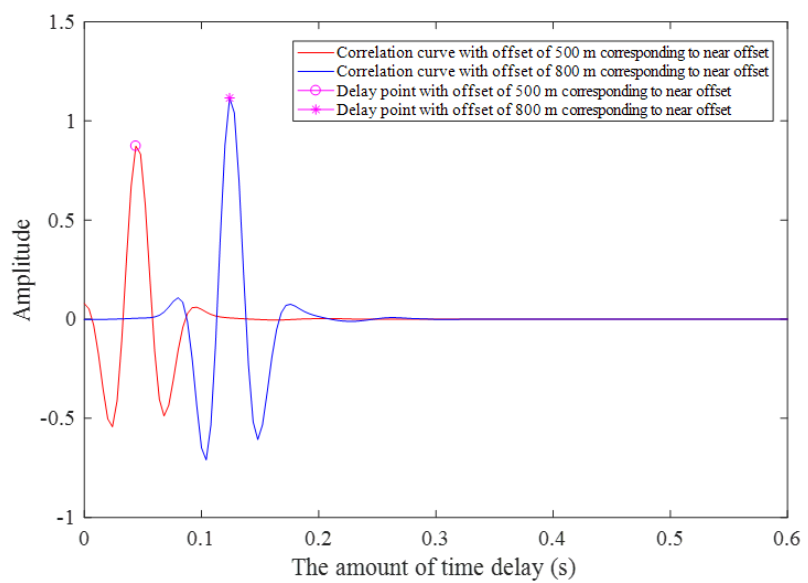

Fig. 4. Cross-correlation processing result.

According to the principle of the cross-correlation function, the corresponding delay amounts at the extreme values of the relevant amplitudes in Figure 3 picked as $0.0440 \mathrm{~s}$ and $0.1240 \mathrm{~s}$, respectively. After comparing the theoretical calculations with the experimental calculations, the difference between the two is $0.0018 \mathrm{~s}$ and $0.0028 \mathrm{~s}$. The percentages of the delays are $4.1 \%$ and $2.3 \%$ respectively, and the corresponding depth scales under the deviation of the delay could be calculated as $3.6144 \mathrm{~m}$ and $5.6224 \mathrm{~m}$. It is known that the source acoustic wave frequency is 40 $\mathrm{Hz}$, so the wavelength in the covering medium on the reflective surface is $50 \mathrm{~m}$, which is much larger than the deviation. Therefore, we could get the conclusion from the simulation experiment that the process of NMO and even the velocity pickup value corresponds with the accuracy requirements. 


\section{Conclusion}

In this paper, based on the time-distance curve equation in reflection seismic exploration and cross-correlation method, the accuracy of the NMO is studied. The amount of NMO is simulated by establishing the forward model and the velocity spectrum analysis. Then, the corresponding reflection seismic data is processed by cross-correlation in MATLAB software to obtain experimental data. Finally, we return to the starting point and compare the theoretical data values with the experimental results to determine whether the accuracy of the amount of NMO and the manual pickup velocity value meet the requirements. Under the reliable simulation experiment, we accurately calculated the accuracy of NMO and obtained a relatively reliable conclusion. Therefore, wo could get the following conclusion that this method demonstrated in this paper provides an effective solution for the precise control of this step of velocity picking. It is helpful for practical application in the field of earth exploration.

\section{References}

1. W.S. Holbrook, P. Paramo, S. Pearse, et al. Thermohaline fine structure in an oceanographic front from seismic reflection profiling. Science, 2003, 301(8): 821.

2. H.Y. Zhou, Y. Jiang. Discuss accuracy question about measuring wavelength for ultrasonic velocity definite using phase method or extreme value method[J]. College Physics, 2007, 26(5): 32-34.

3. X.W. Zhou. The Method of Reflection Seismic Exploration [M]. Beijing: Petroleum Industry Press, 1989.

4. W.C. Yang. Outline of Reflection Seismic Theory [M]. Beijing: Petroleum Industry Press, 2012:2-3.

5. M.M. Abedi, M.A. Riahi. Nonhyperbolic stretchfree normal moveout correction[J]. GEOPHYSICS, 2016, 81(6): U87 - U95.

6. J.J. You, Q. Chen, C.M. Zhao. NMO correction of long-spread seismic events in homogeneous and isotropic me-dia[J]. Progress in Geophysics, 2008, 23:1173-1178. (In Chinese)

7. Y.Y. Zhao, H. Shi, Y.Z. Li. Comparing the accuracy of normal moveout methods in isotropic media[J]. COMPUTING TECHNIQUES FOR GEOPHYSICAL AND EXPLORATION, 2016, 38(5): 626-630.

8. K.M. Wang, L.Y. Lu, Q.H. Liu. Review on the research of earth's attenuation based on the ambient seismic noise cross-correlation function. Progress in Geophysics, 2018, 33(1): 0112-0124. 